\title{
Terahertz Range Surface-Wave Bragg Resonators with Optimized Ratio between Ohmic and Radiative Losses
}

\author{
Andrey M. Malkin, N.S. Ginzburg, S.E. Fil'chenkov, A.S. Sergeev, and V.Yu. Zaslavsky \\ Institute of Applied Physics RAS, Nizhny Novgorod, Russia, malkin@appl.sci-nnov.ru
}

Finite grating area at the surface of a metal plate can form a highly selective surface-wave Bragg resonator which can be used for sensing in the THz range, among other applications [1]. For 1D and 2D structures, we describe surface modes confined at a metallic plate. For a given material conductivity and grating dimensions, an optimum corrugation depth can be determined that provides the maximum value of the fundamental mode quality factor due to the interplay between radiative and Ohmic losses.

Surface electromagnetic waves confined at the interface of metal and dielectric have been widely studied [2] since they possess a number of unique features making them useful for various applications, including antenna applications Raman scattering, etc. Since the evanescent waves are decelerated, Cherenkov devices based on interaction of electron beams with surface modes of the corrugated structures is of special interest in the short wavelength radiation bands, including the terahertz band [3]. These devices allow to solve the problem of longitudinal and transverse mode selection in oversized electrodynamical systems.

In this paper, we consider a periodically corrugated section of a metallic plate as a surface-wave resonator (see Fig.1) in a dielectric media with a permittivity of $\varepsilon$. We obtain the equations of wave coupling with Ohmic losses taken into account and derive an integral equation describing the eigenmodes. Based on this equation, we find the optimum values of the corrugation depth providing the maximum $\mathrm{Q}$ factor of the fundamental mode dependent on the $\varepsilon$. We also discuss the 2D variant of a surface-wave resonator.
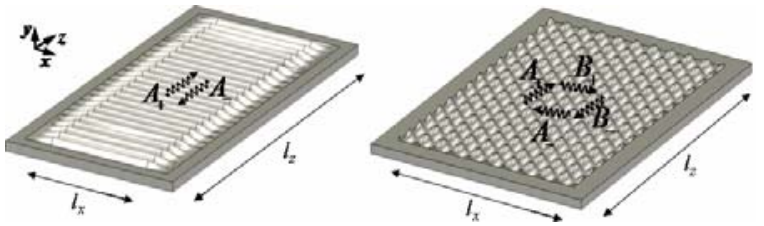

Fig. 1. 1D (left) and 2D (right) surface-wave resonators.

In the case when the depth of sinusoidal corrugation $b(z)=\left(b_{0} / 2\right) \cos \bar{h} z$ is small in the scale of wavelength and of the structure period $d$ the electromagnetic field of the surface mode existing near the $1 \mathrm{D}$ corrugated metal plate can be presented as a sum of two quasi-optical wavebeams with slowly varying amplitudes $A_{ \pm}$which can be characterized by the magnetic field

$$
H_{x}=\operatorname{Re}\left[A_{+}(z, y, t) e^{i\left(\omega_{0} t-h_{0} z\right)}+A_{-}(z, y, t) e^{i\left(\omega_{0} t+h_{0} z\right)}\right],
$$

where $h_{0}=\bar{h} / 2, \omega_{0}=c \varepsilon^{-1 / 2} h_{0}$ is the Bragg frequency. Propagation of electromagnetic field in the vicinity of the Bragg resonance can be described by a system of coupled parabolic wave-beam equations which take into account the diffraction in $\mathrm{y}$ direction [2],

$\pm \frac{\partial A_{ \pm}}{\partial z}+\sqrt{\varepsilon} \frac{\partial A_{ \pm}}{c \partial t}+\sigma \delta(y) A_{ \pm}+\frac{i}{\bar{h}} \frac{\partial^{2} A_{ \pm}}{\partial y^{2}}=i \alpha \delta(y) A_{+}$

At the edges of the corrugation we set non-reflection boundary conditions: $A_{+}(z=0)=0, A_{-}\left(z=l_{z}\right)=0$. Here $\alpha=\bar{h} b_{0} / 8$ is the coupling parameter, $\delta(y)$ is the delta function. Parameter $\sigma=\sqrt{i / 2} \varepsilon k d_{\text {skin }}$ describes the Ohmic losses in the metal corresponding to the Leontovich boundary condition, $d_{\text {skin }}$ is the skin depth of the metal.

Equations (1) applied to a system infinite in $z$ direction lead to the existence of a surface wave $A_{ \pm} \sim e^{i \Gamma z-g_{ \pm} y+i \Omega t}$ with a dispersion law which in the lossless case can be put down as

$$
\varepsilon\left(\frac{\Omega}{c}\right)^{2}-\Gamma^{2}=\bar{h}^{2} \alpha^{4}
$$

Dispersion characteristic corresponding to (2) is shown in the lower part of Fig. 2.

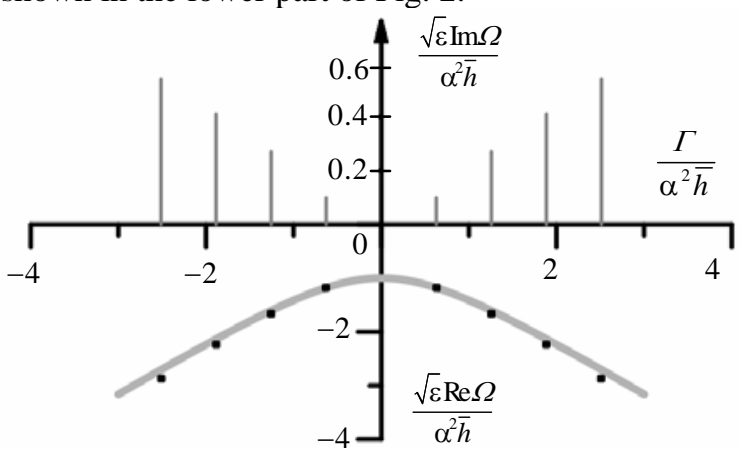

Fig. 2. Dispersion curve of the surface wave. Dots mark the surface-wave resonator modes at $\alpha^{2} \bar{h}_{z}=5$, lines in the upper part indicate the losses of these modes.

In the case when the length $l_{z}$ of the structure is finite, a set of longitudinal modes can be found. Seeking modes of (1) at an eigenfrequency of $\Omega$, and using the Fourier transform, one can obtain an integral equation equivalent to system [5] which possesses an infinite discrete eigenmode spectrum. Frequencies and spatial profiles of eigenmodes can be found numerically. Real parts of the eigenfrequencies of the first several modes are marked with dots close to the dispersion in lower part of Fig.2 for $\alpha^{2} \bar{h} l_{z}=5$ while the imaginary parts of these modes are shown as vertical lines in the upper part of the same figure. It can be seen that the $\operatorname{Im} \Omega$ increases almost linearly. Quality factor $Q=\omega_{0}(2 \operatorname{Im} \Omega)^{-1}$ of the fundamental mode is about 3 times higher than that the next (second mode). It demonstrates the high selective properties of 
surface wave resonator. Making use of the fact that according to the exact solution of (3), the longitudinal field structure of the mode tends to sine shape with increasing parameter $\alpha^{2} \bar{h} l_{z}$, an asymptotic formulas for the fundamental mode eigenfrequency and $\mathrm{Q}$ factor can be obtained in the following form:

$$
\begin{aligned}
& \operatorname{Re} \omega_{1} \approx \frac{c \bar{h}}{\sqrt{\varepsilon}}\left(\frac{1}{2}-\alpha^{2}\left(1+\frac{2 \pi^{2}}{\alpha^{4} \bar{h}^{2} l_{z}^{2}}\right)\right) \\
& Q=\frac{1}{Q_{\text {diff }}^{-1}+Q_{\text {Ohm }}^{-1}}, Q_{\text {diff }}=\frac{\alpha^{4} \bar{h}^{3} l_{z}^{3}}{5 \pi^{2}} ; Q_{O h m}=\frac{2}{\alpha \operatorname{Re} \sigma}
\end{aligned}
$$

Note the sensitivity of the eigenmode position to the value of $\varepsilon$. According to (3), the precision of dielectric sensing by such structure can be roughly estimated as $Q^{-1}$. It is important that the two $Q$ terms in (3) demonstrate different behavior at varying parameter $\alpha$ that is proportional to the corrugation depth $b_{0}$. Total Q factor has a maximum in its $\alpha$ dependence for each $l_{z}$. This maximum can be found from expressions (4) as

$$
Q_{\max }=\left(\frac{2^{4}}{5^{6} \pi^{2}} \frac{l_{z}^{3}}{\bar{h} \varepsilon^{4} d_{\text {skin }}^{4}}\right)^{1 / 5} \approx 0.16 \frac{l_{z}^{3 / 5}}{\bar{h}^{1 / 5} \varepsilon^{4 / 5} d_{\text {skin }}^{4 / 5}}
$$

reached at

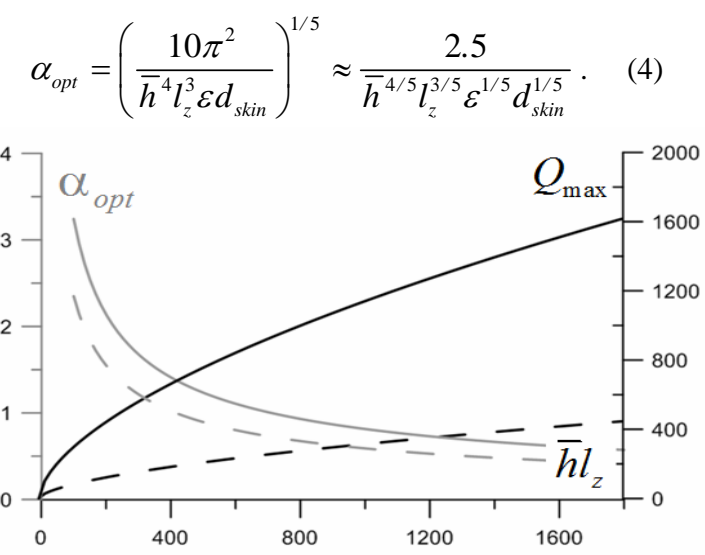

Fig. 3. Optimal $\alpha$ (gray) and maximum Q (black) and vs. length of the resonator. Solid lines correspond to copper structure in vacuum, dashed lines correspond $\varepsilon=5$.

The optimum $\mathrm{Q}$ and $\alpha$ values vs. length of the resonator are depicted in Fig.3 for a structure with a Bragg frequency of about $1 \mathrm{THz}(\mathrm{d}=150 \mu \mathrm{m})$ made of copper $\left(d_{\text {skin }} \approx 0.065 \mu \mathrm{m}\right.$ ) for a structure in vacuum (solid lines) and in a dielectric with $\varepsilon=5$. According to (4), in this parameter area the small corrugation approximation is validated for structures of several dozen periods or longer. For instance, for a structure with a length of 100 periods $\left(\bar{h}_{z} \approx 630\right.$, see dotted line in Fig.5), a corrugation depth of $1 / 10$ wavelength provides an optimal $\mathrm{Q}$ factor of about 1000 for copper at normal temperature. In a dielectric with $\varepsilon=5$, Q value is much lower while the optimum corrugation depth remains almost the same.

Theory of 1D surface-wave resonators given above is applicable only at moderate (in the sense of Fresnel scales) transverse (x) sizes of the system, as it describes, in fact, a single transverse mode approximation. At larger $\mathrm{x}$-dimensions of the system, various transverse modes with close $\mathrm{Q}$ factors would compete with each other. In [5], 2D surface-wave resonators were introduced to provide a means for synchronization of radiation from wide electron beams into the evanescent wave. For a 2D resonator (see Fig.1) with a corrugation of

$$
l(z, x)=l_{2 D} / 4\left[\cos \left(\bar{h}_{2 D}(z-x)\right)+\cos \left(\bar{h}_{2 D}(z+x)\right)\right],
$$

we use the time-domain simulation to find the fundamental mode. In this case, four partial quasi-optical wave-beams take part in formation of a surface mode, two of which $\left(A_{ \pm}\right)$are propagating in $\pm z$ directions and the other two $\left(B_{ \pm}\right)$are propagating in $\pm x$ directions. For 2D Bragg structures, Bragg frequency and wavenumber are defined as $\omega_{0}=c h_{0}, h_{0}=\bar{h}_{2 D}$.

Wavebeam coupling is described by the following system of equations [5]:

$$
\begin{aligned}
& \pm \frac{\partial A_{ \pm}}{\partial z}+\frac{\partial A_{ \pm}}{c \partial t}+\sigma \delta(y) A_{ \pm}+\frac{i}{2 k} \frac{\partial^{2} A_{ \pm}}{\partial y^{2}}=i \alpha_{2 D} \delta(y)\left(B_{+}+B_{-}\right), \\
& \pm \frac{\partial B_{ \pm}}{\partial x}+\frac{\partial B_{ \pm}}{c \partial t}+\sigma \delta(y) B_{ \pm}+\frac{i}{2 k} \frac{\partial^{2} B_{ \pm}}{\partial y^{2}}=i \alpha_{2 D} \delta(y)\left(A_{+}+A_{-}\right) .
\end{aligned}
$$

Here $\alpha_{2 D}=\bar{h}_{2 D} l_{2 D} / 8$. To complete the 3D nonstationary model, we also set an "ideal metal" condition $\partial A_{ \pm} / \partial y=0, \partial B_{ \pm} / \partial y=0$ at some point $y=l_{y}$ with $l_{y}$ much larger than the scale of decay of the fields. These equations were simulated with random initial conditions set at $t=0$ for the parameters of $\alpha_{2 D}^{2} h_{0} l_{z}=4.4, \alpha_{2 D}^{2} h_{0} l_{x}=3.14$. In simulation, a single mode with highest quality factor survived at $\alpha_{2 D}^{2} \omega_{0} t>100$. This fundamental mode is confined at the corrugation and possesses a bell-shaped structure in (z-x) plane. $\mathrm{Q}$ factor of this mode is about $15 \alpha^{-2}$. Thus we have shown that a 2D surface-wave resonator with shallow corrugation is a highly selective structure with respect to three coordinates.

This work was supported by RSF grant No. 1879-10252.

\section{References}

1. B. Ng, J. Wu, S.M. Hanham, et al., Spoof Plasmon Surfaces: A Novel Platform for THz Sensing // Advanced Optical Materials 1(8), 2013.

2. W.L. Barnes, A. Dereux, and T.W. Ebbesen, Surface plasmon subwavelength optics // Nature, v. 424, pp. 824-830, 2003.

3. N.S. Ginzburg, A.M. Malkin, A.S. Sergeev, V.Yu. Zaslavsky, Quasi-optical theory of relativistic submillimeter surface-wave oscillators // Appl. Phys. Lett. 2011, v. 99, p. 121505.

4. N.S. Ginzburg, A.M. Malkin, A.S. Sergeev, V.Yu. Zaslavsky, Powerful surface-wave oscillators with twodimensional periodic structures // Appl. Phys. Lett. 2012, v. 100, p. 143510 .

5. N.S. Ginzburg, A.M. Malkin, A.S. Sergeev, S.E. Fil'chenkov, V.Yu. Zaslavsky, "Highly selective surface-wave resonators for terahertz frequency range formed by metallic Bragg gratings", // Phys. Lett. A, v. 382, p. 925, 2018. 